\title{
Implementation of Condition Equation Model in Geodetic Observation: A Case Study of Circular Reservoir Structure
}

\author{
Oladosu S. O. ${ }^{1, *}$, Okonofua S. E. ${ }^{2}$ and Ehigiator-Irughe R. ${ }^{3}$ \\ ${ }^{1,2,3}$ Department of Geomatics, Faculty of Environmental Sciences, University of Benin, Nigeria \\ Corresponding Author: *olushola.oladosu@uniben.edu
}

https://doi.org/10.36263/nijest.2019.02.0120

\begin{abstract}
In engineering projects involving the construction of aboveground storage tank big enough to retain and/or accommodate large quantities of petroleum products such as crude oil and condensate, mathematical reduction of obtained field data using condition equation method is always appropriate for an onward monitoring of those structures. This paper demonstrates how condition equation method can be used to adjust geodetic surveying measurements in relation to aboveground storage tank. Accuracy is in the order of $\sigma_{a}=5.66 e-4$ and $\sigma_{b}=1.113 e-3$ while $\sigma_{\text {smax }}$ and $\sigma_{\text {smin }}$ are 0.0522 and -0.0511 respectively. The results obtained revealed that the method can be satisfactorily implemented for aboveground circular reservoir storage tank structural modelling and monitoring for a similar scenario.
\end{abstract}

Keywords: Crude oil, Condition equation, Observation, Storage tank

\subsection{Introduction}

Aboveground storage tanks (ASTs), are commonly used to store large quantities of petroleum products such as crude oil or condensate. Reservoirs used by oil companies in Nigeria are basically cylindrical in shape. In most cases due to age, non-uniformity of foundation, geological conditions loading and offloading, crude oil temperature, primary and secondary settlement of sediments results in radial deformation or out of roundness (Ehigiator-Irughe et al., 2012).

Aboveground storage tanks (ASTs) situated at the Forcados terminal were constructed between 1967 and 1970. Ten (10) of these crude oil storage reservoirs each spanning around $22 \mathrm{~m}$ in height and of diameter $76.2 \mathrm{~m}$ are presently in operation. Figures 1 and 2 show the study area and a typical example of the kind of cylindrical tank about which data collection were made.

Periodic monitoring of these tanks has been suggested by Ehigiator-Irughe and Ehigiator (2010). The structural integrity of these reservoirs has been of major concern to both local community and environmentalists especially in Niger delta area of the country. Although API 653 remain the industry standard relative to reservoir inspection and maintenance, the frequency of testing and inspection can also be affected by various State and Local regulations (Ehigiator-Irughe and Ehigiator, 2010).

The schedule of this inspection process depend on a number of factors as noted by Ehigiator-Irughe $e t$ al. (2011) which include the age of tank, their proximity to groundwater, the leak records, the date of the last integrity test, the construction material used, the product stored, soil condition, among others. Storage tanks (reservoirs) at Forcados farm are bounded with a bound wall measuring $250 \mathrm{~m}$ by $150 \mathrm{~m}$ and at a height of $12 \mathrm{~m}$ respectively. These bound walls are intended to accommodate as well as contain any accidental spill that may result from failure of any of the reservoir as a temporary remedial means.

The objective of this paper is to determine the vertical movement or shift in the position of the tanks using a precise geodetic levelling instrument DL101C and to establish horizontal controls around the monitoring site by adopting a Total station (Sokkia SET1130R) instrument. Prior to the period of 
carrying out this work, no such equipment and monitoring operation exercise of that nature has been adopted for the same purpose at the study area.

\subsection{Materials and Methods}

\subsection{Study area}

The study area (Forcados) terminal is located in Burutu Local Government Area of Delta State, Nigeria, forming boundary with the Bight of Benin by the Atlantic Ocean with coordinates (759624.59 m E, $591614.74 \mathrm{~m} \mathrm{~N}$ and $760885.71 \mathrm{~m} \mathrm{E}, 590242.51 \mathrm{~m} \mathrm{~N}$ ) in UTM Zone 31 (Figures 1 and 2).

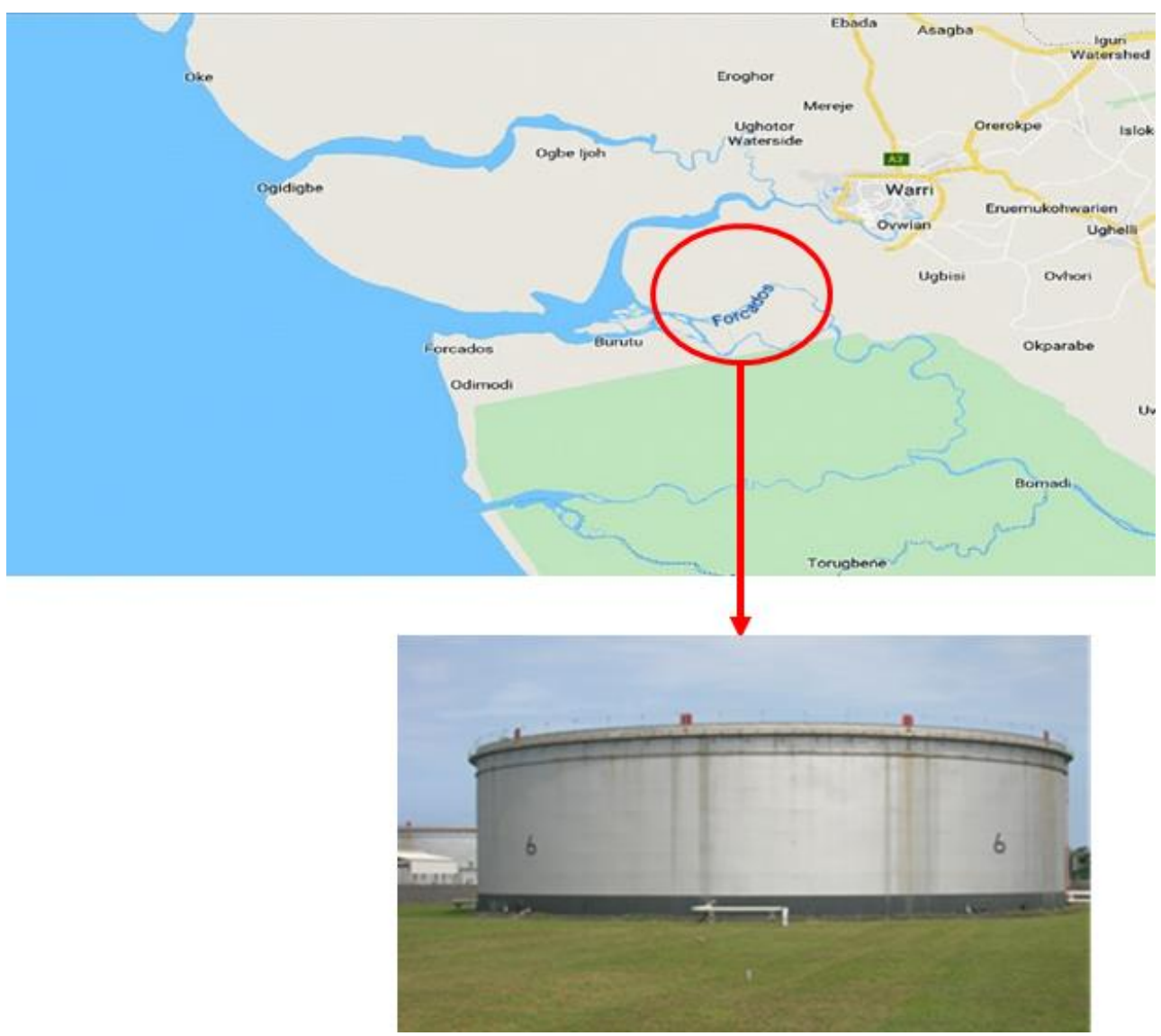

Figure 1: Location of the study area and a typical crude oil tank

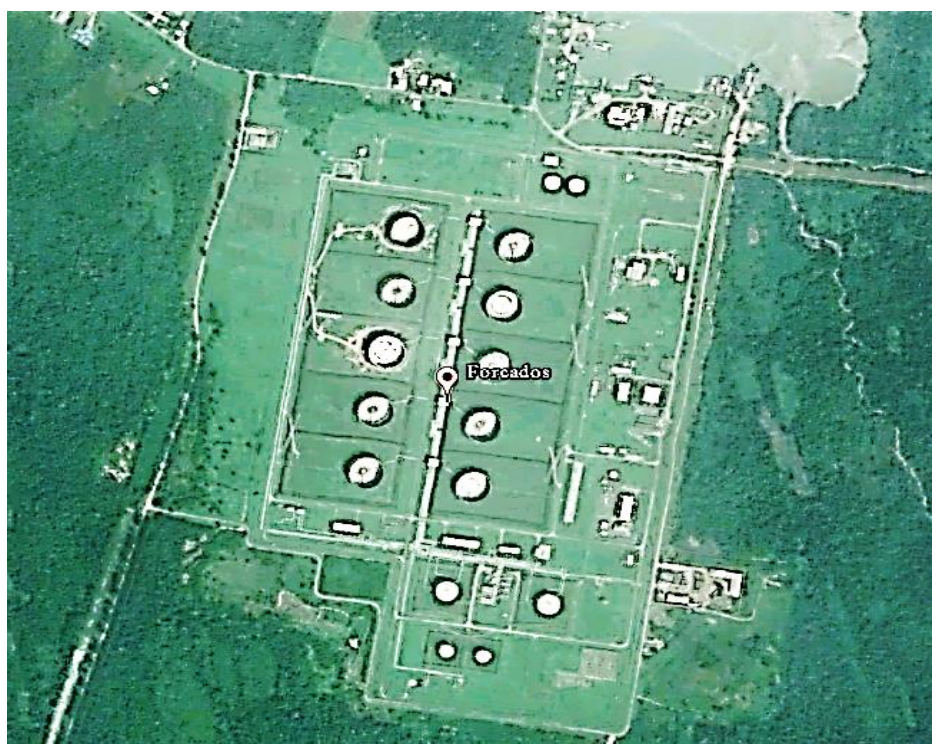

Figure 2: Screen shot of the study area. 


\subsection{Materials}

The materials used in this work include steel tape, Total station (Sokkia SET1130R), precise geodetic level DL101C, Staff, Computer set and accessories, etc. The circular cross section of each storage tanks was divided into 16 monitoring points distributed around the tanks. These monitoring points were fixed at equal intervals on the outer face of the tanks and placed at $2 \mathrm{~m}$ high from the tanks base. These monitoring points were established in such a way that they remained fixed throughout the period of observation.

In order to ensure the reliability of the monitoring points, they were tied to higher order control points established in some stable ground and some distances away from the tanks. The total station instrument used for the measurement was mounted on these control points and observation taken to the monitoring station in the tank face. Measurements were made using a combination of angular and linear intersection with the aid of the total station instrument to reflectors held on the studs.

\subsection{Results}

Figure 3 is a typical model of the levelling networks adopted for the measurement of the height/elevation of the 16 studs, while Table 1 shows the difference in elevation obtained during the exercise.

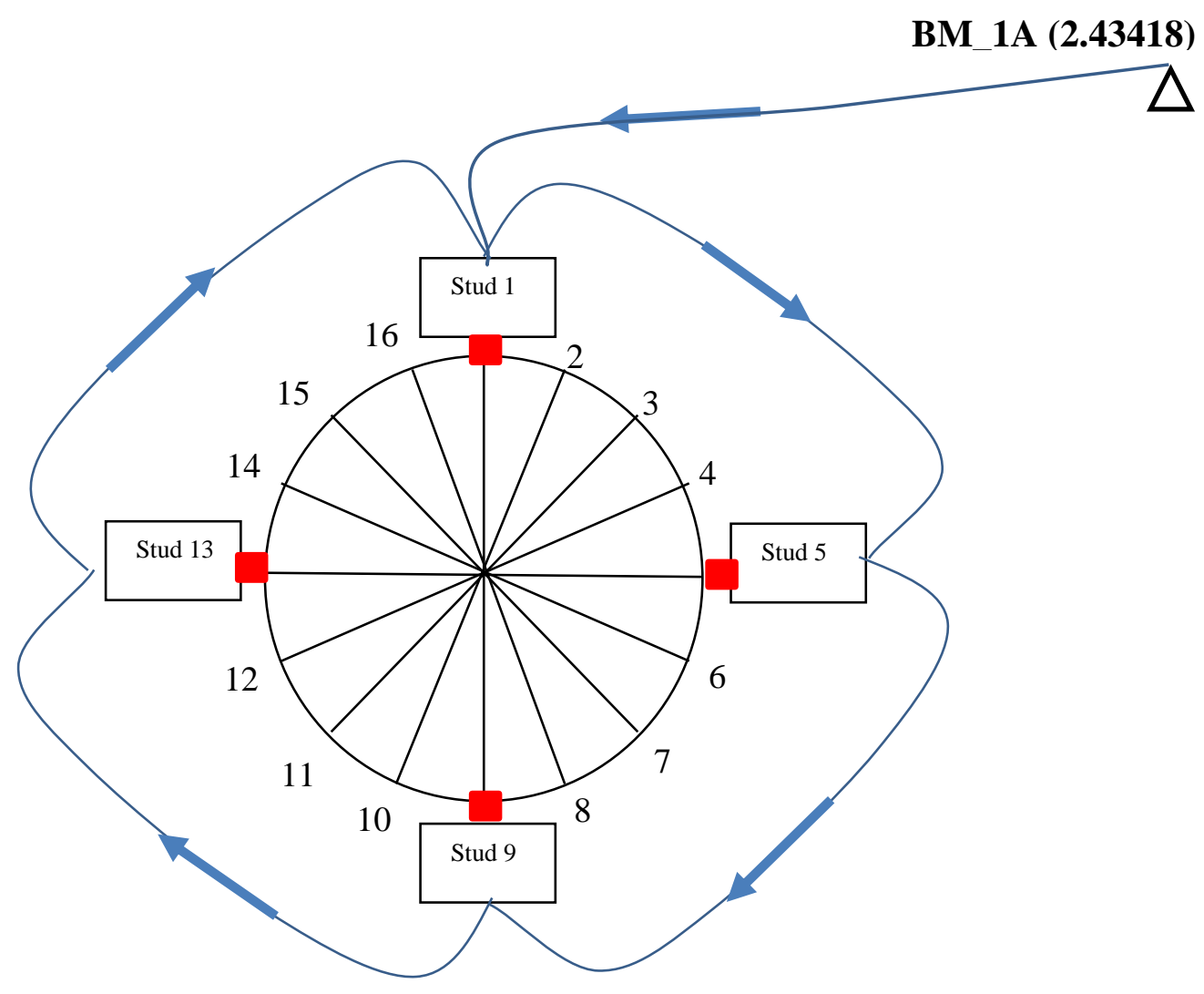

Figure 3: Levelling observation networks 
Table 1: Differences in elevation before adjustment

\begin{tabular}{|l|l|}
\hline STUDS & Diff. Elev. $(\mathrm{m})$ \\
\hline STUD1 & $\Delta \mathrm{h} 1=1.02036$ \\
\hline STUD2 & $\Delta \mathrm{h} 2=0.01157$ \\
\hline STUD3 & $\Delta \mathrm{h} 3=-0.00026$ \\
\hline STUD4 & $\Delta \mathrm{h} 4=0.00432$ \\
\hline STUD5 & $\Delta \mathrm{h} 5=-0.00363$ \\
\hline STUD6 & $\Delta \mathrm{h} 6=0.00352$ \\
\hline STUD7 & $\Delta \mathrm{h} 7=0.00482$ \\
\hline STUD8 & $\Delta \mathrm{h} 8=0.00406$ \\
\hline STUD9 & $\Delta \mathrm{h} 9=-0.00143$ \\
\hline STUD10 & $\Delta \mathrm{h} 10=0.00085$ \\
\hline STUD11 & $\Delta \mathrm{h} 11=-0.00238$ \\
\hline STUD12 & $\Delta \mathrm{h} 12=0.00209$ \\
\hline STUD13 & $\Delta \mathrm{h} 13=-0.00615$ \\
\hline STUD14 & $\Delta \mathrm{h} 14=-0.00290$ \\
\hline STUD15 & $\Delta \mathrm{h} 15=-0.00524$ \\
\hline STUD16 & $\Delta \mathrm{h} 16=-0.00151$ \\
\hline BM_1A & -1.02469 \\
\hline
\end{tabular}

\subsection{Implementation of condition equation}

The method of condition equations otherwise known as the method of correlates was described by (Ayeni, 2001; Ghilani, 2006) as one which establishes a set of equations which must be satisfied by the true values of observations, given certain geometrical conditions or physical laws of nature imposed by the configuration of the problem. This is because the true values of observations exist only in the metaphysical/supernatural world. It is only practicable to set up condition equations which relate together some adjusted (that is, most probable value of observations). Using the condition equation as follows:

$$
\begin{aligned}
& B M_{-} 1 A+\left(V_{1}+\Delta h_{1}\right)+\left(V_{2}+\Delta h_{2}\right)+\left(V_{3}+\Delta h_{3}\right)+\left(V_{4}+\Delta h_{4}\right)+\left(V_{5}+\Delta h_{5}\right)+\left(V_{6}+\Delta h_{6}\right) \\
& +\left(V_{7}+\Delta h_{7}\right)+\left(V_{8}+\Delta h_{8}\right)+\left(V_{9}+\Delta h_{9}\right)+\left(V_{10}+\Delta h_{10}\right)+\left(V_{11}+\Delta h_{11}\right)+\left(V_{12}+\Delta h_{12}\right) \\
& +\left(V_{13}+\Delta h_{13}\right)+\left(V_{14}+\Delta h_{14}\right)+\left(V_{15}+\Delta h_{15}\right)+\left(V_{16}+\Delta h_{16}\right)+\left(V_{17}+\Delta h_{17}\right)=B M_{-} 1 A
\end{aligned}
$$

Substituting the value of the BM height we have:

$$
\begin{aligned}
& 2.43418+\left(\mathrm{V}_{1}+\Delta \mathrm{h}_{1}\right)+\left(\mathrm{V}_{2}+\Delta \mathrm{h}_{2}\right)+\left(\mathrm{V}_{3}+\Delta \mathrm{h}_{3}\right)+\left(\mathrm{V}_{4}+\Delta \mathrm{h}_{4}\right)+\left(\mathrm{V}_{5}+\Delta \mathrm{h}_{5}\right)+\left(\mathrm{V}_{6}+\Delta \mathrm{h}_{6}\right)+ \\
& \left(\mathrm{V}_{7}+\Delta \mathrm{h}_{7}\right)+\left(\mathrm{V}_{8}+\Delta \mathrm{h}_{8}\right)+\left(\mathrm{V}_{9}+\Delta \mathrm{h}_{9}\right)+\left(\mathrm{V}_{10}+\Delta \mathrm{h}_{10}\right)+\left(\mathrm{V}_{11}+\Delta \mathrm{h}_{11}\right)+\left(\mathrm{V}_{12}+\Delta \mathrm{h}_{12}\right)+\left(\mathrm{V}_{13}+\Delta \mathrm{h}_{13}\right) \\
& +\left(\mathrm{V}_{14}+\Delta \mathrm{h}_{14}\right)+\left(\mathrm{V}_{15}+\Delta \mathrm{h}_{15}\right)+\left(\mathrm{V}_{16}+\Delta \mathrm{h}_{16}\right)+\left(\mathrm{V}_{17}+\Delta \mathrm{h}_{17}\right)=2.43418 .
\end{aligned}
$$

By substituting the values of $\left(\Delta \mathrm{h}_{\mathrm{i}}\right)$ we have the following:

$$
\begin{aligned}
& V_{1}+V_{2}+V_{3}+V_{4}+V_{5}+V_{6}+V_{7}+V_{8}+V_{9}+V_{10}+V_{11}+V_{12}+V_{13}+V_{14}+V_{15}+V_{16}+V_{17}= \\
& 2.43418-2.43418-1.02036-0.01157+0.00026-0.00432+ \\
& 0.00363-0.00352-0.00482-0.00406+0.00143-0.00085+ \\
& 0.00238-0.00209+0.00615+0.0029+0.00524+0.00151+1.02469 \\
& V_{1}+V_{2}+V_{3}+V_{4}+V_{5}+V_{6}+V_{7}+V_{8}+V_{9}+V_{10}+V_{11}+V_{12}+V_{13}+V_{14}+V_{15}+V_{16}+V_{17} \\
& =0.00340
\end{aligned}
$$

Note $; \mathrm{Vi}=$ Residual, $\Delta$ hi $=$ Difference in elevation, $B M_{-} I A=$ Reference elevation .

The general form of condition equation is given as:

$$
[B] \times[V]=[\Delta]
$$

where $[B]$ is the design Matrix and is given as:

$B=[11111111111111111]$, and $\mathrm{V}=$ the vector of Residuals; $[\Delta]=0.00340$ 


$$
V=\left[\begin{array}{l}
V 1 \\
V 2 \\
V 3 \\
V 4 \\
V 5 \\
V 6 \\
V 7 \\
V 8 \\
V 9 \\
V 10 \\
V 11 \\
V 12 \\
V 13 \\
V 14 \\
V 16 \\
V 17
\end{array}\right]
$$

The solution of the normal equation:

$M=[B] \times[B]^{T}$

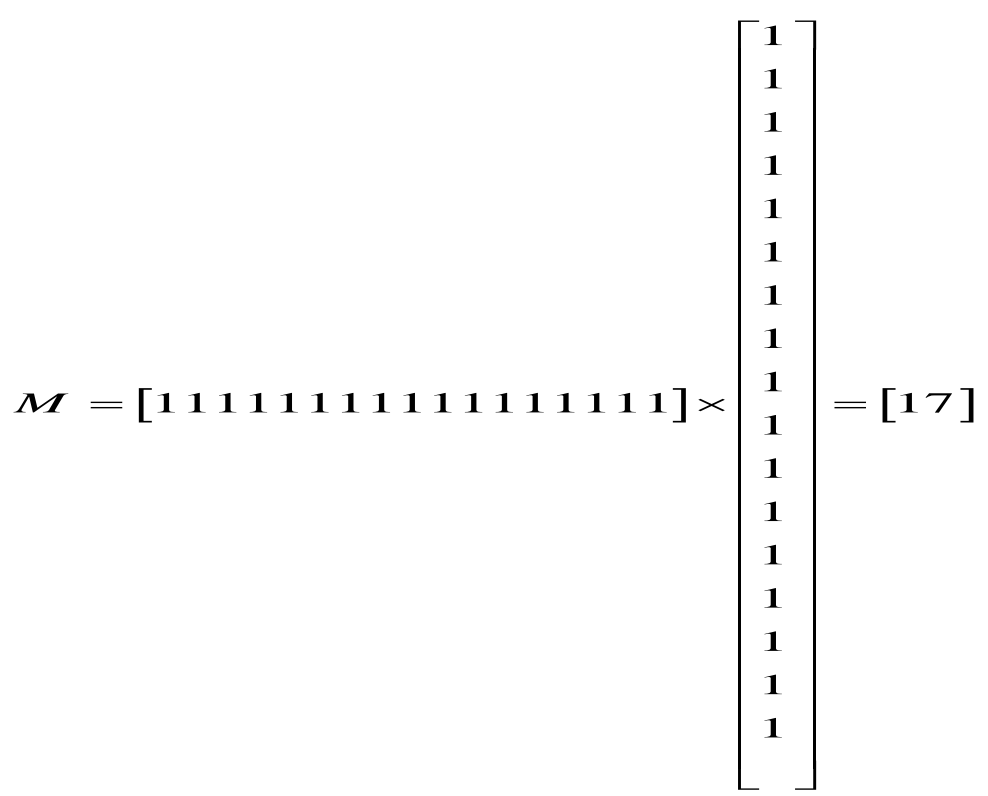

The solution of Lagrange Multiplier [K]:

$$
\begin{aligned}
& K=[M]^{-1} \times \Delta \\
& K=[17]^{-1} \times[0.00340] \\
& K=\left[2.0 \times 10^{-04}\right]
\end{aligned}
$$

The residuals:

$\left[V_{i}\right]=[B]^{T} \times[K]$ 


$$
\left[V_{i}\right]=\left[\begin{array}{c}
2.00 \\
2.00 \\
2.00 \\
2.00 \\
2.00 \\
2.00 \\
2.00 \\
2.00 \\
2.00 \\
2.00 \\
2.00 \\
2.00 \\
2.00 \\
2.00 \\
2.00 \\
2.00 \\
2.00
\end{array}\right] \times 10^{4}
$$

The variance:

$$
\sigma^{2}=\left\{\frac{\left[V_{i}\right]^{T} \times[V]}{r}\right\}
$$

Numbers of unknowns $=16$, numbers of observations $=18$.

Therefore, redundancy (degree of freedom) $=2$,

$$
\begin{aligned}
\sigma^{2}= & \left\{\frac{\left[V_{i}\right]^{T} \times[V]}{2}\right\} \\
& {\left[\begin{array}{l}
2.00 \\
2.00 \\
2.00 \\
2.00 \\
2.00 \\
2.00 \\
2.00 \\
2.00 \\
2.00 \\
2.00 \\
2.00 \\
2.00 \\
2.00 \\
2.00 \\
2.00 \\
2.00 \\
2.00
\end{array}\right] \times 10^{4}\left[\begin{array}{l}
2.00 \\
2.00 \\
2.00 \\
2.00 \\
2.00 \\
2.00 \\
2.00 \\
2.00 \\
2.00 \\
2.00 \\
2.00 \\
2.00 \\
2.00 \\
2.00 \\
2.00 \\
2.00 \\
2.00
\end{array}\right] \times 10^{4} }
\end{aligned}
$$




$$
\sigma^{2}=\left[3.4 \times 10^{-7}\right]
$$

Table 2 shows the adjusted elevations for the sixteen tanks (studs) using condition equation model method respectively.

Table 2: The adjusted elevations

\begin{tabular}{|c|c|c|c|c|c|c|c|}
\hline \multirow{2}{*}{ Station No } & \multirow{2}{*}{$\begin{array}{l}\text { Staff Set - } \\
\text { Up }\end{array}$} & \multicolumn{2}{|c|}{ Staff Readings } & \multirow{2}{*}{$\begin{array}{l}\text { Prov. Rise (+) } \\
\text { Prov. Fall (-) } \\
\end{array}$} & \multirow{2}{*}{$\begin{array}{l}\text { Corr. } \\
\mathrm{mm} \\
\end{array}$} & \multirow{2}{*}{$\begin{array}{l}\text { Final Rise (+) } \\
\text { Final Fall (-) }\end{array}$} & \multirow{2}{*}{$\begin{array}{l}\text { Final Elev. (m) } \\
\text { Datum----- }\end{array}$} \\
\hline & & Back & Fore & & & & \\
\hline & BM_1A & 1.49409 & & & & & 2.43418 \\
\hline $\mathrm{P} 1$ & STUD1 & 0.46644 & 0.47373 & 1.02036 & 0.00020 & 1.02056 & 3.45474 \\
\hline $\mathrm{P} 2$ & STUD2 & 0.55877 & 0.45487 & 0.01157 & 0.00020 & 0.01177 & 3.46651 \\
\hline P3 & STUD3 & 0.56466 & 0.55903 & -0.00026 & 0.00020 & -0.00006 & 3.46645 \\
\hline $\mathrm{P} 4$ & STUD4 & 0.54440 & 0.56034 & 0.00432 & 0.00020 & 0.00452 & 3.47103 \\
\hline P5 & STUD5 & 0.59716 & 0.54803 & -0.00363 & 0.00020 & -0.00343 & 3.46760 \\
\hline P6 & STUD6 & 0.37493 & 0.59364 & 0.00352 & 0.00020 & 0.00372 & 3.47475 \\
\hline P7 & STUD7 & 0.62290 & 0.37011 & 0.00482 & 0.00020 & 0.00502 & 3.47605 \\
\hline P8 & STUD8 & 0.63397 & 0.61884 & 0.00406 & 0.00020 & 0.00426 & 3.47529 \\
\hline P9 & STUD9 & 0.32043 & 0.63540 & -0.00143 & 0.00020 & -0.00123 & 3.46980 \\
\hline P10 & STUD10 & 0.37890 & 0.31958 & 0.00085 & 0.00020 & 0.00105 & 3.47208 \\
\hline P11 & STUD11 & 0.45879 & 0.38128 & -0.00238 & 0.00020 & -0.00218 & 3.46885 \\
\hline $\mathrm{P} 12$ & STUD12 & 0.49455 & 0.45670 & 0.00209 & 0.00020 & 0.00229 & 3.47332 \\
\hline $\mathrm{P} 13$ & STUD13 & 0.26153 & 0.50070 & -0.00615 & 0.00020 & -0.00595 & 3.46508 \\
\hline $\mathrm{P} 14$ & STUD14 & 0.39577 & 0.26443 & -0.00290 & 0.00020 & -0.00270 & 3.46833 \\
\hline $\mathrm{P} 15$ & STUD15 & 0.81228 & 0.40101 & -0.00524 & 0.00020 & -0.00504 & 3.46599 \\
\hline \multirow[t]{2}{*}{$\mathrm{P} 16$} & STUD16 & 0.50664 & 0.81379 & -0.00151 & 0.00020 & -0.00131 & 3.46468 \\
\hline & BM_1A & & 1.53133 & -1.02469 & 0.00020 & -1.02449 & 2.43418 \\
\hline
\end{tabular}

Table 3 is showing the studs and their adjusted difference in elevation in relation to the bench (reference) station used for the observation.

Table 3: Studs and their respective adjusted difference in elevation

\begin{tabular}{|l|l|}
\hline STUDS & Adjusted Diff. Elev. $(\mathrm{m})$ \\
\hline STUD1 & $\Delta \mathrm{h} 1=1.02056$ \\
\hline STUD2 & $\Delta \mathrm{h} 2=0.01177$ \\
\hline STUD3 & $\Delta \mathrm{h} 3=-0.00006$ \\
\hline STUD4 & $\Delta \mathrm{h} 4=0.00452$ \\
\hline STUD5 & $\Delta \mathrm{h} 5=-0.00343$ \\
\hline STUD6 & $\Delta \mathrm{h} 6=0.00372$ \\
\hline STUD7 & $\Delta \mathrm{h} 7=0.00502$ \\
\hline STUD8 & $\Delta \mathrm{h} 8=0.00426$ \\
\hline STUD9 & $\Delta \mathrm{h}=-0.00123$ \\
\hline STUD10 & $\Delta \mathrm{h} 10=0.00105$ \\
\hline STUD11 & $\Delta \mathrm{h} 11=-0.00218$ \\
\hline STUD12 & $\Delta \mathrm{h} 12=0.00229$ \\
\hline STUD13 & $\Delta \mathrm{h} 13=-0.00595$ \\
\hline STUD14 & $\Delta \mathrm{h} 14=-0.00270$ \\
\hline STUD15 & $\Delta \mathrm{h} 15=-0.00504$ \\
\hline STUD16 & $\Delta \mathrm{h} 16=-0.00131$ \\
\hline BM_1A & -1.02469 \\
\hline
\end{tabular}




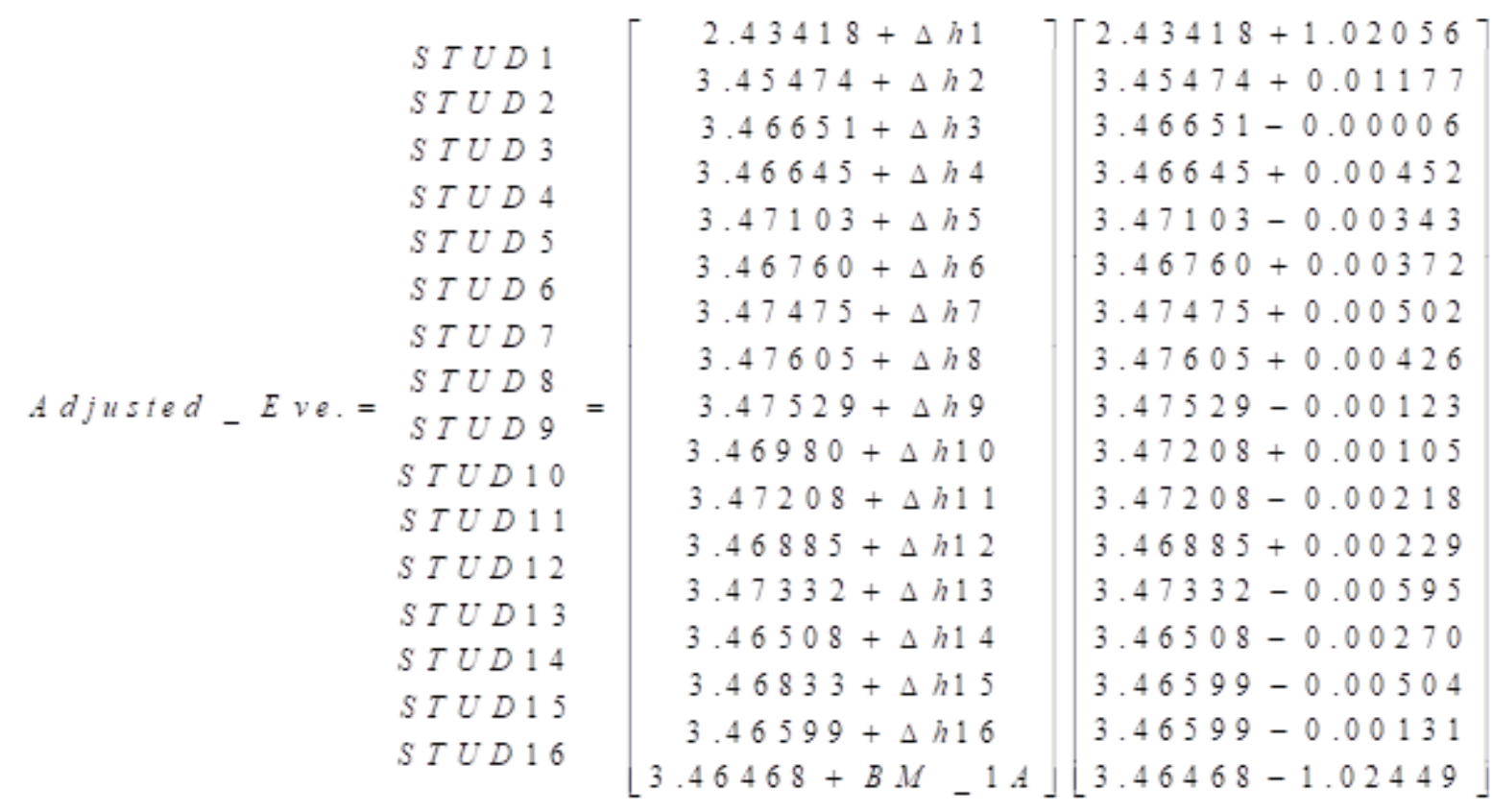

Ajusted_Evelation $(m)=\left[\begin{array}{lllll}3.4 & 5 & 4 & 7 & 4 \\ 3.4 & 6 & 6 & 5 & 1 \\ 3.4 & 6 & 6 & 4 & 5 \\ 3.4 & 7 & 1 & 0 & 3 \\ 3.4 & 6 & 7 & 6 & 0 \\ 3.4 & 7 & 4 & 7 & 5 \\ 3.4 & 7 & 6 & 0 & 5 \\ 3.4 & 7 & 5 & 2 & 9 \\ 3.4 & 6 & 9 & 8 & 0 \\ 3.4 & 7 & 2 & 0 & 8 \\ 3.4 & 6 & 8 & 85 \\ 3.4 & 7 & 3 & 3 & 2 \\ 3.4 & 6 & 5 & 0 & 8 \\ 3.4 & 6 & 8 & 3 & 3 \\ 3.4 & 6 & 5 & 9 & 9 \\ 3.4 & 6 & 4 & 6 & 8 \\ 2.4 & 3 & 4 & 1 & 8\end{array}\right]$

The Variance Covariance (V-C) matrix is given as:

$\left.\begin{array}{l}C_{L}=\sigma^{2}[1-T] W^{-1} \\ T=W^{-1} B^{T} M^{-1} B \\ C_{L}=\sigma^{2}\left[1-B^{T} M^{-1} B\right]\end{array}\right\}$

Since $W^{-1}=1$ 
$C_{L}=$

\begin{tabular}{|c|c|c|c|c|c|c|c|c|c|c|c|c|c|c|c|c|}
\hline & $=07$ & & & & & & & & & & & & & & & \\
\hline$E-0$ & $=07$ & & & 007 & & -07 & $=-07$ & -07 & & & 2E-07 & & & & & \\
\hline$[-07$ & $=07$ & $E-07$ & & -07 & & E-07 & E-07 & -07 & & & $E-07$ & & & & & \\
\hline 2E-07 & $=0 /$ & & $2 E-07$ & & & {$[-07$} & & .07 & & & 01 & & & & & \\
\hline$E-07$ & $=-07$ & & & & & & & $2 E-07$ & & & & & & & & \\
\hline 2E-07 & E-07 & $E-07$ & E-OT & $E-07$ & $: 07$ & E-07 & $E-07$ & $2 \mathrm{E}-07$ & $=07$ & E- -07 & $E-07$ & $E-07$ & E-07 & $E-07$ & $2 E-0\}$ & \\
\hline 2E-07 & E-07 & E-07 & $2 E-07$ & E-07 & E-07 & ZE-07 & $3.2 E-07$ & $2 \mathrm{E}-07$ & 2E-07 & $2 E-07$ & 2E-07 & 2E-07 & $2 E-07$ & LE-UI & $\langle E-U|$ & \\
\hline $2 E-07$ & & & & & & & & & & & & & & & & \\
\hline $2 \mathrm{E}-07$ & $E-07$ & $E-07$ & $2 E-07$ & $E-07$ & $E-07$ & ZE-07 & $3.2 E-07$ & $2 E-07$ & 2E-07 & $2 \mathrm{EE}-07$ & $E-07$ & $2 \mathrm{E}-07$ & 2E-07 & $E=0$ & $2 \mathrm{E}-\mathrm{C}$ & \\
\hline 2E-07 & E-07] & {$[-07$} & $2 E-07$ & E-07 & E-07 & 3.2E-07 & $3.2 E-07$ & $3.2 E-07$ & 2E-07 & $3.2 E-07$ & 2E-07 & .2E-07 & $2 \mathrm{E}-$ & 2E-07 & $2 \mathrm{E}-$ & \\
\hline$E-07$ & E-07 & 01 & E-07) & -01 & E-0) & $10-0$ & $3.2 E-07$ & 2E-07 & $\langle E-0|$ & 3.2E-07 & J.LLEVI & $2,-0)$ & & & $22-01$ & \\
\hline 2-07 & E-07 & $E-07$ & $2 \mathrm{EE}-07$ & .07 & $E-07$ & 2E-07 & $3.2 E-07$ & $2 \mathrm{E}-07$ & $3.2 E-07$ & $2 E-07$ & $: 07$ & $2 E-07$ & 3.2E- & $2 F_{-}$ & $3.2 \mathrm{E}-$ & \\
\hline $2 E-07$ & $E-07$ & $E-07$ & 3.2E-07 & $E-07$ & $E-07$ & 2E-07 & $3.2 E-07$ & 2E-07 & $2 \mathrm{E}-07$ & $3.2 E-07$ & $E-07$ & $2 \mathrm{EE}-07$ & -07 & E-07 & I) F- & \\
\hline 2E-07 & E-07 & E-07 & 2E-07 & E-07 & E-07 & 3. LEt-U & 3.2E-07 & 2E-07] & $2 E-0$ & $2 \mathrm{EE}-07$ & $=01$ & 2E-07 & .2E-0I & $\angle E-0)$ & .2E-C & \\
\hline E-07 & t.UT & $1=0 /$ & $2 E-07$ & 2E-07 & $\mid=-0)$ & $3.2 E-07$ & $3.2 E-07$ & $0 /$ & $3.2 E-01$ & $2 E-07$ & & {$[-01$} & II & {$[=-0,1$} & $E=-0$ & \\
\hline$[-07$ & {$[-07$} & & $2 E-07$ & & & & & & & & & & & 1 & 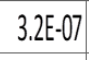 & \\
\hline $2 \mathrm{E}-07$ & E-07 & $=07$ & $2 E-07$ & E-07 & $\mathrm{E}-07$ & 3.2E-07 & .2E-0 & 2E-07 & 2E-07 & $3.2 E-07$ & $3.2 E-07$ & $2 \mathrm{EE}-07$ & 2E-07 & 2E-07 & $3.2 E-07$ & \\
\hline
\end{tabular}

Constructing Jacobian Matrix of post adjustment, we have:

$$
J_{I 17 \times 17}=\left[\begin{array}{cccc}
\frac{\partial H_{1}}{\partial \Delta h_{1}} & \frac{\partial H_{1}}{\partial \Delta h_{2}} & \frac{\partial H_{1}}{\partial \Delta h_{3}} \ldots & \frac{\partial H_{1}}{\partial \Delta h_{n}} \\
\frac{\partial H_{2}}{\partial \Delta h_{1}} & \frac{\partial H_{2}}{\partial \Delta h_{2}} & \frac{\partial H_{2}}{\partial \Delta h_{3}} \ldots & \frac{\partial H_{2}}{\partial \Delta h_{n}} \\
\frac{\partial H_{3}}{\partial \Delta h_{1}} & \frac{\partial H_{3}}{\partial \Delta h_{2}} & \frac{\partial H_{2}}{\partial \Delta h_{3}} \ldots & \frac{\partial H_{3}}{\partial \Delta h_{n}} \\
\cdot & \vdots & \ddots & \vdots \\
\cdot & \cdot & \cdots & \cdot \\
\frac{\partial \dot{H}_{u}}{\partial \Delta h_{1}} & \frac{\partial \dot{H}_{u}}{\partial \Delta h_{2}} & \frac{\partial \dot{H}_{u}}{\partial \Delta h_{3}} & \frac{\partial \dot{H}_{u}}{\partial \Delta h_{n}}
\end{array}\right]
$$

$$
J_{I 17 \times 17=}=\left[\begin{array}{ccccccccccccccccc}
1 & 0 & 0 & 0 & 0 & 0 & 0 & 0 & 0 & 0 & 0 & 0 & 0 & 0 & 0 & 0 & 0 \\
1 & 1 & 0 & 0 & 0 & 0 & 0 & 0 & 0 & 0 & 0 & 0 & 0 & 0 & 0 & 0 & 0 \\
1 & 1 & -1 & 0 & 0 & 0 & 0 & 0 & 0 & 0 & 0 & 0 & 0 & 0 & 0 & 0 & 0 \\
1 & 1 & -1 & 1 & 0 & 0 & 0 & 0 & 0 & 0 & 0 & 0 & 0 & 0 & 0 & 0 & 0 \\
1 & 1 & -1 & 1 & -1 & 0 & 0 & 0 & 0 & 0 & 0 & 0 & 0 & 0 & 0 & 0 & 0 \\
1 & 1 & -1 & 1 & -1 & 1 & 0 & 0 & 0 & 0 & 0 & 0 & 0 & 0 & 0 & 0 & 0 \\
1 & 1 & -1 & 1 & -1 & 1 & 1 & 0 & 0 & 0 & 0 & 0 & 0 & 0 & 0 & 0 & 0 \\
1 & 1 & -1 & 1 & -1 & 1 & 1 & 1 & 0 & 0 & 0 & 0 & 0 & 0 & 0 & 0 & 0 \\
1 & 1 & -1 & 1 & -1 & 1 & 1 & 1 & -1 & 0 & 0 & 0 & 0 & 0 & 0 & 0 & 0 \\
1 & 1 & -1 & 1 & -1 & 1 & 1 & 1 & -1 & 1 & 0 & 0 & 0 & 0 & 0 & 0 & 0 \\
1 & 1 & -1 & 1 & -1 & 1 & 1 & 1 & -1 & 1 & -1 & 0 & 0 & 0 & 0 & 0 & 0 \\
1 & 1 & -1 & 1 & -1 & 1 & 1 & 1 & -1 & 1 & -1 & 1 & 0 & 0 & 0 & 0 & 0 \\
1 & 1 & -1 & 1 & -1 & 1 & 1 & 1 & -1 & 1 & -1 & 1 & -1 & 0 & 0 & 0 & 0 \\
1 & 1 & -1 & 1 & -1 & 1 & 1 & 1 & -1 & 1 & -1 & 1 & -1 & -1 & 0 & 0 & 0 \\
1 & 1 & -1 & 1 & -1 & 1 & 1 & 1 & -1 & 1 & -1 & 1 & -1 & -1 & -1 & 0 & 0 \\
1 & 1 & -1 & 1 & -1 & 1 & 1 & 1 & -1 & 1 & -1 & 1 & -1 & -1 & -1 & -1 & 0
\end{array}\right]
$$

Post Adjustment V-C Matrix is the V-C matrix $\boldsymbol{C}_{\boldsymbol{H}}$ of the adjusted benchmarks $H$ and is computed as follows: Because the products of both the Jacobian and V-C matrices are large, the first 3 rows and 
first 3 columns on both V-C Matrix and the Jacobian Matrix, will be used for post adjustment V-C matrix as presented below;

$$
\mathrm{C}_{\mathrm{H}}=J_{H} C_{L} J_{H}{ }^{T} \mathrm{~s}
$$

Where:

$$
\begin{aligned}
\boldsymbol{J}_{\boldsymbol{H}} & =\frac{\partial G(L)}{\partial L}=\left[\begin{array}{cccccc}
1 & 0 & 0 & 0 & 0 & 0 \\
1 & 1 & 0 & 0 & 0 & 0 \\
1 & 1 & -1 & 0 & 0 & 0 \\
1 & 1 & -1 & 1 & 0 & 0 \\
1 & 1 & -1 & 1 & -1 & 0 \\
1 & 1 & -1 & 1 & -1 & 1
\end{array}\right] \\
C L & =\left[\begin{array}{llllll}
3.20 & 3.20 & 3.20 & 3.20 & 3.20 & 3.20 \\
3.20 & 3.20 & 3.20 & 3.20 & 3.20 & 3.20 \\
3.20 & 3.20 & 3.20 & 3.20 & 3.20 & 3.20 \\
3.20 & 3.20 & 3.20 & 3.20 & 3.20 & 3.20 \\
3.20 & 3.20 & 3.20 & 3.20 & 3.20 & 3.20 \\
3.20 & 3.20 & 3.20 & 3.20 & 3.20 & 3.20
\end{array}\right] \times 10^{-7}
\end{aligned}
$$

The V-C matrix $\boldsymbol{C}_{\boldsymbol{H}}$ of the adjusted benchmarks $H$ is then computed thus;

$$
C_{H}=\left[\begin{array}{llllll}
0.0320 & 0.0640 & 0.0320 & 0.0640 & 0.0320 & 0.0640 \\
0.0640 & 0.1280 & 0.0640 & 0.1280 & 0.0640 & 0.1280 \\
0.0320 & 0.0640 & 0.0320 & 0.0640 & 0.0320 & 0.0640 \\
0.0640 & 0.1280 & 0.0640 & 0.1280 & 0.0640 & 0.1280 \\
0.0320 & 0.0640 & 0.0320 & 0.0640 & 0.0320 & 0.0640 \\
0.0640 & 0.1280 & 0.0640 & 0.1280 & 0.0640 & 0.1280
\end{array}\right] \times 10^{-5}
$$

accuracy $:\left(\sigma_{a}\right)=\sqrt{0.032 \times 10^{-5}}=5.66 \times 10^{-4}$

$$
\begin{aligned}
& \text { accuracy }:\left(\sigma_{b}\right)=\sqrt{0.128 \times 10^{-5}}=1.113 \times 10^{-3} \\
& \text { and } \\
& \left(\sigma_{a b}\right)=0.0640 \times 10^{-5}
\end{aligned}
$$

$$
2 t=\tan ^{-1}\left\{\frac{2 \sigma_{a b}}{\sigma_{b}^{2}-\sigma_{a}^{2}}\right\}
$$

$$
\begin{aligned}
& 2 t=\tan ^{-1}\left\{\frac{2 \times 0.0640}{0.1280-0.0320}\right\} \times 10^{-5} \\
& 2 t=1.333
\end{aligned}
$$$$
2 t=53^{\circ} 07^{\prime} 46^{\prime \prime}
$$$$
\mathrm{t}=26^{\circ} 33^{\prime} 53^{\prime},
$$ 
The problem now is to develop a new covariance matrix from existing $Q$ matrix which removes correlation between the unknowns; and the determination of semi-major axis and the semi-minor axis of error ellipse, from which we have:

$$
\left.\begin{array}{l}
C_{z z}=R \times C_{L L} \times R^{T} \\
R=\left[\begin{array}{cc}
\sin (t) & \cos (t) \\
-\cos (t) & \sin (t)
\end{array}\right] \\
C_{z z}=\left[\begin{array}{cc}
\sin (t) & \cos (t) \\
-\cos (t) & \sin (t)
\end{array}\right]\left[\begin{array}{ll}
\sigma_{a}^{2} & \sigma_{a b} \\
\sigma_{b a} & \sigma_{b}^{2}
\end{array}\right]\left[\begin{array}{ll}
\sin (t) & -\cos (t) \\
\cos (t) & \sin (t)
\end{array}\right]
\end{array}\right]
$$

The semi-major axis of error ellipse was obtained as $\sigma_{s \max }=7.65 \times 10^{-4}$, while the semi-minor axis was obtained as $\sigma_{s \min }=6.27 \times 10^{-4}$

\subsection{Discussion}

The method of condition equation adopted in this work showed a promising result and can be adopted in similar scenarios. Accuracy obtained is in the order of $\sigma_{a}=5.66 \times 10^{-4}$ and $\sigma_{b}=1.113 \times 10^{-3}$ before $\sigma_{a b}=0.0640 \times 10^{-5}$ for the adjusted bench marks heights while the error ellipse are $\sigma_{\text {smax }}=7.65 \times 10^{-4}$ and $\sigma_{s \min }=6.27 \times 10^{-4}$ respectively, representing insignificant error and acceptable values. However, the values were obtained after the application of new covariance matrix from existing $Q$ matrix which removes correlation between the unknowns. The determination of semi-major axis and the semi-minor axis error ellipse gave $\sigma=5.83 \times 10^{-4}$ which is a way to ascertain the magnitude of error at a station.

\subsection{Conclusion}

In this work, attempt has been made to implement condition equation method in circular reservoir structures (above ground tanks) relying on geodata acquired and further preparation for onward analysis of the shift experienced by these structures at the Forcados crude oil tank farm. Since each condition equation must satisfy certain geometric conditions or physical laws. Suggestion is to adopt the process in similar scenarios across the country where such structures exist in order to buttress on the safety of lives of the inhabitants living close to their location.

\section{References}

Ayeni, O. O. (2001). Statistical adjustment and analysis of data. A Manual, in the Department of Surveying \& Geoinformatics, Faculty of Engineering, University of Lagos, Nigeria, 2001. 
Ehigiator - Irughe, R. and Ehigiator, M. O. (2010). Estimation of the centre coordinates and radius of Forcados Oil Tank from Total Station data using least square Analysis. International Journal of Pure and Applied Sciences. A pan - African Journal Series.

Ehigiator - Irughe, R. Ashraf, A. B., Ehiorobo, J. O. and Ehigiator, O. M. (2011). Modification of Geodetic Methods for Determining the Monitoring Station Coordinates on the Surface of Cylindrical Oil Storage Tank. Research Journal of Engineering and Applied Sciences (RJEAS), 1(1), pp. 58 - 63. A United State Academy publications USA.

Ehigiator-Irughe, R., Ehiorobo, J. O., Ashraf, A. B. and Ehigiator, M. O. (2012). Determination of the Ovality of Crude Oil Storage Tanks using Least Squares. Advanced Materials Research, 367, pp. 475483, Trans Tech Publications, Switzerland doi:10.4028/www.scientific.net/AMR.367.475.

Ghilani, C. D. and Wolf, P. R. (2006). Adjustment Computations: Spatial Data Analysis. Fourth Edition. John Wiley \& Sons, Inc. ISBN: 978-0-471-69728-2. 